\title{
CONTROLLABILITY PROPERTIES FOR THE ONE-DIMENSIONAL HEAT EQUATION UNDER MULTIPLICATIVE OR NONNEGATIVE ADDITIVE CONTROLS WITH LOCAL MOBILE SUPPORT *,**
}

\author{
Luis Alberto Fernández ${ }^{1}$ And Alexander Yuri Khapalov ${ }^{2}$
}

\begin{abstract}
We discuss several new results on nonnegative approximate controllability for the onedimensional Heat equation governed by either multiplicative or nonnegative additive control, acting within a proper subset of the space domain at every moment of time. Our methods allow us to link these two types of controls to some extend. The main results include approximate controllability properties both for the static and mobile control supports.
\end{abstract}

Mathematics Subject Classification. 35K05, 35K20, 93B05.

Received February 21, 2011. Revised November 7, 2011.

Published online March 27, 2012.

\section{Problem Setting}

In this paper we study the approximate controllability properties of the one dimensional Heat equation governed either by an additive nonnegative locally distributed control or by a multiplicative control of local support. For simplicity, we will assume that the space domain is the interval $(0,1)$. More precisely, we consider the following two Dirichlet problems:

$$
\begin{cases}y_{t}(x, t)=y_{x x}(x, t)+u(x, t) \chi_{\omega(t)}(x), & \text { in } Q_{T}=(0,1) \times(0, T), \\ y(0, t)=y(1, t)=0, & \text { in }(0, T), \\ y(x, 0)=y_{0}(x), & \text { in }(0,1),\end{cases}
$$

and

$$
\begin{cases}y_{t}(x, t)=y_{x x}(x, t)+v(x, t) y(x, t) \chi_{\omega(t)}(x), & \text { in } Q_{T} \\ y(0, t)=y(1, t)=0, & \text { in }(0, T), \\ y(x, 0)=y_{0}(x), & \text { in }(0,1),\end{cases}
$$

Keywords and phrases. Parabolic equation, approximate controllability, multiplicative controls, nonnegative locally distributed controls.

* The work of the first author was partially supported by the Spanish Ministry of Science and Innovation under grant MTM2008 - 04206.

** The work of the second author was supported in part by the NSF Grant DMS-1007981.

1 Departamento de Matemáticas, Estadística y Computación, Avda. de los Castros, s/n, Universidad de Cantabria, 39005

Santander, Spain. lafernandez@unican.es

2 Department of Mathematics, Washington State University, Pullman, 99164-3113 WA, USA. khapala@math.wsu.edu 
where $y_{0} \in L_{+}^{2}(0,1)=\left\{\phi \in L^{2}(0,1): \phi(x) \geq 0\right.$ a.e. in $\left.(0,1)\right\}$, with $y_{0} \not \equiv 0$ for $(S 2)$. In the above, $u$ is the additive locally distributed control, while $v$ is the multiplicative (or bilinear) control (in their respective systems), each one supported at each time $t$ in a proper subinterval $\omega(t)$ of $[0,1]$. We will assume that

$$
\omega(t)=(r(t), r(t)+l),
$$

where the length $l \in(0,1)$ is fixed and $r:[0, T] \longrightarrow[0,1-l]$ is a function that defines the position of the support at time $t$. We call the support $\omega(t)$ static if it does not change in time and mobile if it does. The symbol $\chi_{\omega(t)}$ denotes the characteristic function of $\omega(t)$ (i.e. $\chi_{\omega(t)}(x)=1$, when $x \in \omega(t)$ and $\chi_{\omega(t)}(x)=0$, for $x \notin \omega(t)$ ). In the sequel, we will denote by $\tilde{y}_{y_{0}, u, r}$ the solution of system $(S 1)$ and by $y_{y_{0}, v, r}$ the solution of system $(S 2)$. By the linearity of $(S 1)$ it is clear that $\tilde{y}_{y_{0}, u, r}=\tilde{y}_{y_{0}}+\tilde{y}_{0, u, r}$, where $\tilde{y}_{y_{0}}$ denotes the unique solution of $(S 1)$ with $u=0$. If the subinterval $\omega(t)=\omega$ for all $t$, (i.e. it is static), we will use the notation $\tilde{y}_{y_{0}, u, \omega}$ instead of $\tilde{y}_{y_{0}, u, r}$ (resp., $y_{y_{0}, v, \omega}$ instead of $y_{y_{0}, v, r}$ ).

The control $u$ in $(S 1)$ represents an external source acting within $\omega(t)$. In turn, the multiplicative control $v$ in $(S 2)$ can be viewed as controlling the reaction rate of the process $(S 2)$ within its support.

We will investigate how rich the sets of solutions to the above controlled systems are at the fixed final time $T>0$. To that end, let us introduce the following notations:

$$
\begin{aligned}
& F_{1, y_{0}, m b}^{+}(T)=\left\{\tilde{y}_{y_{0}, u, r}(x, T): x \in(0,1), u \in L_{+}^{2}\left(Q_{T}\right) \text { and } r \in P C[0, T]\right\}, \\
& F_{2, y_{0}, m b}(T)=\left\{y_{y_{0}, v, r}(x, T): x \in(0,1), v \in L^{\infty}\left(Q_{T}\right) \text { and } r \in P C[0, T]\right\},
\end{aligned}
$$

where $P C[0, T]$ denotes the set of functions $r:[0, T] \longrightarrow[0,1-l]$ such that $r$ is piecewise constant with (at most) a finite number of discontinuities and $L_{+}^{2}\left(Q_{T}\right)=\left\{\phi \in L^{2}\left(Q_{T}\right): \phi(x, t) \geq 0\right.$ a.e. in $\left.Q_{T}\right\}$.

System $(S 1)$ is said to be approximately nonnegative controllable at time $T>0$ if for any given $y_{0} \in L_{+}^{2}(0,1)$, the set $F_{1, y_{0}, m b}^{+}(T)$ is dense in $\tilde{y}_{y_{0}}(\cdot, T)+L_{+}^{2}(0,1)$. Taking into account the linearity of the PDE, it is quite easy to verify

$$
F_{1, y_{0}, m b}^{+}(T)=\tilde{y}_{y_{0}}(\cdot, T)+F_{1,0, m b}^{+}(T) .
$$

Hence, the approximate nonnegative controllability at time $T>0$ is clearly equivalent to the density of $F_{1,0, m b}^{+}(T)$ in $L_{+}^{2}(0,1)$. Analogously, system $(S 2)$ is said to be approximately nonnegative controllable at time $T>0$ if for any given $y_{0} \in L_{+}^{2}(0,1), y_{0} \not \equiv 0$, the set $F_{2, y_{0}, m b}(T)$ is dense in $L_{+}^{2}(0,1)$. For $y_{0} \equiv 0$, by the uniqueness of solution for the system $(S 2)$, it is obvious that $F_{2,0, m b}(T)=\{0\}$ and the aforementioned density property in $L_{+}^{2}(0,1)$ clearly does not hold.

Formally, it is apparent that the PDE in $(S 2)$ can be obtained from the corresponding one in $(S 1)$ by setting

$$
v(x, t)=\frac{u(x, t)}{y(x, t)} .
$$

This identity will be a key point to get our results. Of course, these two problems are not equivalent in the sense that in $(S 1)$ the control $u$ is always nonnegative, while $v$ in $(S 2)$ can be negative as well, thus providing a "qualitatively richer" set of solutions for $(S 2)$ compared to that of $(S 1)$.

When the support of the control is the whole space domain, it was shown in [8] that the set

$$
F_{1, y_{0}}^{+}(T)=\left\{\tilde{y}_{y_{0}, u, \omega}(x, T): \quad x \in(0,1), u \in L_{+}^{2}\left(Q_{T}\right), \quad \omega \equiv(0,1), \quad \forall t \in(0, T)\right\}
$$

is dense in $\tilde{y}_{y_{0}}(\cdot, T)+L_{+}^{2}(0,1)$. Furthermore, it was shown in [9-11] that the corresponding property also holds for the system $(S 2)$ : i.e., the set

$$
F_{2, y_{0}}(T)=\left\{y_{y_{0}, v, \omega}(x, T): x \in(0,1), v \in L^{\infty}\left(Q_{T}\right), \quad \omega \equiv(0,1), \quad \forall t \in(0, T)\right\}
$$


is dense in $L_{+}^{2}(0,1)$. In fact, these results remain true for more general linear and semilinear parabolic equations in several space dimensions. In this paper, however, we are specifically interested in the case when $\omega(t)$ is a proper subinterval of $(0,1)$ at every moment of time.

Let us emphasize that the multiplicative controllability problem is of nonlinear nature, even in the case of the linear equation like $(S 2)$, because the mapping $v \rightarrow y_{y_{0}, v, \omega}$ is highly nonlinear: for instance, when $\omega=(0,1)$ and $v$ is constant, it is well known that $y_{y_{0}, v, \omega}(x, t)=\tilde{y}_{y_{0}}(x, t) \mathrm{e}^{-v t}$. This means that we cannot use here the traditional duality argument, which is the typical strategy to approach controllability problems in the case of linear PDE with additive controls.

Among earlier works on the controllability of linear PDE by means of bilinear controls we refer to the pioneering work [3] by Ball et al. The paper [18] further explored the ideas of [3].

An extensive and thorough bibliography on controllability of bilinear ODE is available. Let us just mention the survey [1]. Research in this area was seemingly originated in the 60's, on the one hand, by the works of Kucera, who linked this area to the Lie Algebra approach, and, on the other hand, by the works of Rink and Mohler [25] (see also [17]), who pursued the qualitative approach and numerous applications.

For recent works on global controllability of parabolic and hyperbolic linear and semilinear PDE we refer to $[6,11,13-15]$ and specially to the monograph [16] and the references therein. In the last years, intensive research has been carried out concerning the Schrödinger equation (quantum control), see for instance $[5,7,8,10]$.

We also refer to the works $[4,20,21]$ (see also the references therein) on the issue of optimal bilinear control for various PDE. A closely related issue is stabilization by means of bilinear controls, see [2,23].

\section{MAIN RESULTS}

First of all, we will show that for static subdomain the above controllability property is out of the question for both systems $(S 1)$ and $(S 2)$.

Theorem 2.1 (Lack of controllability for static support). Let us assume that $y_{0} \in L_{+}^{2}(0,1), y_{0} \not \equiv 0$, and $\omega$ is a fixed proper subinterval of $(0,1)$. Then, for any $T>0$, the sets

$$
F_{1,0, \omega}^{+}(T)=\left\{\tilde{y}_{0, u, \omega}(x, T): x \in(0,1), u \in L_{+}^{2}\left(Q_{T}\right), \omega(t) \equiv \omega, \quad \forall t \in(0, T)\right\},
$$

and

$$
F_{2, y_{0}, \omega}(T)=\left\{y_{y_{0}, v, \omega}(x, T): x \in(0,1), v \in L^{\infty}\left(Q_{T}\right), \quad \omega(t) \equiv \omega, \quad \forall t \in(0, T)\right\}
$$

are not dense in $L_{+}^{2}(0,1)$.

Nevertheless, we can prove (in a constructive way) the following "regional' controllability result.

Theorem 2.2 (Regional nonnegative controllability). Let us assume that $\omega$ is a fixed proper subinterval of $(0,1)$. Then, for any $T>0$, the set

$$
F_{1,0, \omega}^{+}(T)_{\mid \omega}=\left\{\tilde{y}_{0, u, \omega}(x, T): x \in \omega, u \in L_{+}^{2}\left(Q_{T}\right), \quad \omega(t) \equiv \omega, \quad \forall t \in(0, T)\right\},
$$

is dense in $L_{+}^{2}(\omega)$.

If the control support is allowed to move with an additional piecewise constant control $r(t)$, defining its position at time $t$, we have the following global controllability result.

Theorem 2.3 (Approximate controllability with mobile support). Let us assume that $y_{0} \in L_{+}^{2}(0,1), y_{0} \not \equiv 0$. Then, for any $T>0$, the sets $F_{1,0, m b}^{+}(T)$ and $F_{2, y_{0}, m b}(T)$ are dense in $L_{+}^{2}(0,1)$. 
In the proof of Theorem 2.1 we have faced with the following system:

$$
\begin{cases}y_{t}(x, t)=y_{x x}(x, t), & \text { in } Q_{T}, \\ y(0, t)=u_{0}(t), & \text { in }(0, T), \\ y(1, t)=u_{1}(t), & \text { in }(0, T), \\ y(x, 0)=y_{0}(x), & \text { in }(0,1),\end{cases}
$$

with $u_{0}$ and $u_{1}$ acting as boundary controls, meanwhile $y_{0}$ is fixed.

If we introduce the set

$$
F_{y_{0}, b}(T)=\left\{y_{y_{0}, u_{0}, u_{1}}(x, T): x \in(0,1), u_{0}, u_{1} \in L_{+}^{\infty}(0, T)\right\}
$$

where $y_{y_{0}, u_{0}, u_{1}}$ denotes the unique solution of $(S 3)$ defined by transposition (see [22], pp. 194-197), we have derived a related result on the lack of nonnegative approximate boundary controllability with nonnegative boundary controls, in the following terms:

Theorem 2.4. Let us assume that $y_{0} \in L_{+}^{2}(0,1)$. Then, for any $T>0$, the set $F_{y_{0}, b}(T)$ is not dense in $\tilde{y}_{y_{0}}(\cdot, T)+L_{+}^{2}(0,1)$.

An essential tool for obtaining these results are the classical Maximum Principles for parabolic equations (both in the weak and strong form). Since they will be cited very frequently along the paper and there are different versions depending on the framework, we recall them here:

Theorem 2.5 (Maximum principles). Let us assume that $y_{0} \in L^{2}(0,1), u \in L^{2}\left(Q_{T}\right)$ and $v \in L^{\infty}\left(Q_{T}\right)$. Let $y \in L^{2}\left(0, T ; H^{1}(0,1)\right) \cap C\left([0, T] ; L^{2}(0,1)\right)$ be a solution of the problem

$$
\begin{cases}y_{t}(x, t)=y_{x x}(x, t)+v(x, t) y(x, t)+u(x, t), & \text { in } Q_{T}, \\ y(x, 0)=y_{0}(x), & \text { in }(0,1) .\end{cases}
$$

(i) If $y_{0} \geq 0$ in $(0,1), u \geq 0$ in $Q_{T}, y(0, t)$ and $y(1, t) \geq 0$ in $(0, T)$, then $y(x, t) \geq 0$ a.e. in $Q_{T}$;

(ii) if $u \equiv 0, v \leq 0$ in $Q_{T}, y(0, \cdot), y(1, \cdot) \in L^{\infty}(0, T)$ and $y_{0} \in L^{\infty}(0,1)$, then $y(x, t) \leq K$ a.e. in $Q_{T}$, with

$$
K=\max \left\{\left\|(y(0, \cdot))^{+}\right\|_{L^{\infty}(0, T)},\left\|(y(1, \cdot))^{+}\right\|_{L^{\infty}(0, T)},\left\|\left(y_{0}\right)^{+}\right\|_{L^{\infty}(0,1)}\right\}
$$

where $z^{+}=\max \{z, 0\}$;

(iii) (Strong) If $y \in L^{2}\left(0, T ; H_{0}^{1}(0,1)\right), y_{0} \geq 0$ in $(0,1)\left(y_{0} \not \equiv 0\right), u \geq 0$ in $Q_{T}, v=v(x) \leq 0$ in $(0,1)$, then $y(x, t)>0$ in $(0,1)$ for all $t \in(0, T]$.

For the proofs, we refer (among others) to [19], Chapter III, Section 7, pages 181-191, and [24], pages 21-23. See also [19], Chapter I, Section 2, pages 11-25, for related results in the classical framework.

\section{Proof of Theorems 2.1, 2.2 And 2.4}

We begin with the proof of Theorem 2.1 for system $(S 1)$. Our goal is to construct a simple explicit counterexample.

\subsection{Proof of Theorem 2.1 for system $(S 1)$}

Since $u \in L_{+}^{2}\left(Q_{T}\right)$, it is well known (see for instance [19], Thm. 4.2, p. 160), that each solution $\tilde{y}_{0, u, \omega}$ belongs to $L^{2}\left(0, T ; H_{0}^{1}(0,1)\right) \cap C\left([0, T] ; L^{2}(0,1)\right)$. Moreover, due to the Theorem 2.5-(i)

$$
\tilde{y}_{0, u, \omega}(x, t) \geq 0 \text { a.e. } x \in(0,1), \forall t \in[0, T] .
$$

Consequently, $F_{1,0, \omega}^{+}(T) \subset L_{+}^{2}(0,1)$. We will show that $F_{1,0, \omega}^{+}(T)$ is not dense in $L_{+}^{2}(0,1)$. 
Since $\omega$ is a fixed proper subinterval of $(0,1)$, there will exist a sufficiently large natural number $m \geq 2$ such that $\omega \subset\left(\frac{1}{m}, 1\right)$. Let us fix that $m$ for the rest of the proof. It is now straightforward to check that if

$$
p(x, t)=\varphi(x) \exp \left(\frac{m^{2} \pi^{2}(t-T)}{(m-1)^{2}}\right)
$$

with

$$
\varphi(x)= \begin{cases}\sin (m \pi x), & x \in\left[0, \frac{1}{m}\right), \\ (1-m) \sin \left(\frac{\pi(m x-1)}{m-1}\right), & x \in\left[\frac{1}{m}, 1\right],\end{cases}
$$

$p \in C^{2}\left(\overline{Q_{T}}\right)$ and it is the unique classical solution of the adjoint problem

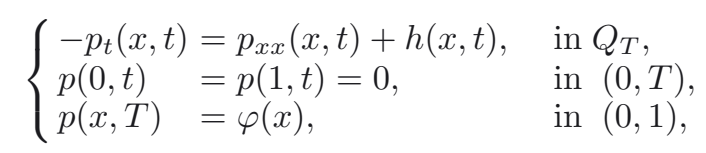

where

$$
h(x, t)= \begin{cases}\frac{(m-2) m^{3} \pi^{2}}{(m-1)^{2}} \sin (m \pi x) \exp \left(\frac{m^{2} \pi^{2}(t-T)}{(m-1)^{2}}\right), & x \in\left[0, \frac{1}{m}\right), \\ 0, & x \in\left[\frac{1}{m}, 1\right] .\end{cases}
$$

Furthermore,

$$
\begin{gathered}
h(x, t) \geq 0 \quad \text { in } \overline{Q_{T}} \\
p(x, t) \leq 0 \quad \text { in }\left[\frac{1}{m}, 1\right] \times[0, T] .
\end{gathered}
$$

Let us now pick up the state in $L_{+}^{2}(0,1)$

$$
y_{d}(x)=\max \{\varphi(x), 0\} .
$$

We will see that $y_{d}$ is "unachievable". Arguing by contradiction, let us suppose that there exists a sequence $\left\{u_{k}\right\}_{k=1}^{\infty} \subset L_{+}^{2}\left(Q_{T}\right)$ such that:

$$
\tilde{y}_{0, u_{k}, \omega}(\cdot, T) \longrightarrow y_{d} \quad \text { in } L^{2}(0,1) \text { as } k \rightarrow+\infty .
$$

Multiplying $(S 1)$ (with $u=u_{k}, \omega(t)=\omega$ and $y_{0}=0$ ) by $p$, further integration by parts, combined with (3.1)-(3.3) provides:

$$
\begin{aligned}
\int_{0}^{1} \tilde{y}_{0, u_{k}, \omega}(x, T) \varphi(x) \mathrm{d} x & \leq \int_{0}^{1} \tilde{y}_{0, u_{k}, \omega}(x, T) \varphi(x) \mathrm{d} x+\int_{Q_{T}} h(x, t) \tilde{y}_{0, u_{k}, \omega}(x, t) \mathrm{d} x \mathrm{~d} t \\
& =\int_{\omega \times(0, T)} u_{k}(x, t) p(x, t) \mathrm{d} x \mathrm{~d} t \leq 0 \quad \forall k .
\end{aligned}
$$

Thus, we arrive at the contradiction

$$
0<\int_{0}^{\frac{1}{m}}(\sin (m \pi x))^{2} \mathrm{~d} x=\int_{0}^{1} y_{d}(x) \varphi(x) \mathrm{d} x=\lim _{k} \int_{0}^{1} \tilde{y}_{0, u_{k}, \omega}(x, T) \varphi(x) \mathrm{d} x \leq 0 .
$$

This ends the proof of Theorem 2.1 for system $(S 1)$. 


\subsection{Proof of Theorem 2.2}

It can be obtained by a simple modification of the proof given in [9] for the case $\omega=(0,1)$. Nonetheless, the argument used there does not provide any insight on what controls are used to achieve the desirable steering. Therefore, here we give a different constructive proof of Theorem 2.2, through Fourier series expansions, which can be used to prove the aforementioned result in [9] as well and will also serve us as a tool to prove the Theorem 2.3 below.

Consider any element $y_{d} \in L_{+}^{2}(\omega)$. Extend it by zero to the whole interval $(0,1)$ and, for simplicity of notations, denote the resulting function by $y_{d}$ again. Clearly, it lies in $L_{+}^{2}(0,1)$. Given any $\delta \in(0, T)$, we define

$$
u_{\delta}(x, t)= \begin{cases}0, & t \in[0, T-\delta) \\ \frac{1}{\delta} y_{d}(x), & t \in[T-\delta, T]\end{cases}
$$

Taking now advantage of the Fourier series expansion, we know

$$
y_{d}(x)=2 \sum_{k=1}^{\infty} a_{k} \sin (k \pi x), \quad \text { in } L^{2}(0,1),
$$

with $a_{k}=\int_{0}^{1} y_{d}(x) \sin (k \pi x) \mathrm{d} x$.

The classical method of separation of variables provides us the following expression for the corresponding solution of $(S 1)$ with $y_{0}=0$

$$
\tilde{y}_{0, u_{\delta}, \omega}(x, t)= \begin{cases}0, & t \in[0, T-\delta], \\ \frac{2}{\delta} \sum_{k=1}^{\infty} \frac{1-\mathrm{e}^{-\pi^{2} k^{2}(t-T+\delta)}}{\pi^{2} k^{2}} a_{k} \sin (k \pi x), & t \in[T-\delta, T] .\end{cases}
$$

Hence, we derive that

$$
\left\|\tilde{y}_{0, u_{\delta}, \omega}(\cdot, T)-y_{d}\right\|_{L^{2}(0,1)}^{2}=2 \sum_{k=1}^{\infty} a_{k}^{2}\left(\frac{1-\mathrm{e}^{-\pi^{2} k^{2} \delta}}{\pi^{2} k^{2} \delta}-1\right)^{2} .
$$

Let us show that the right-hand side of (3.8) tends to zero as $\delta \rightarrow 0^{+}$. To that end, we introduce the auxiliary function

$$
\psi(r)=\left(\frac{1-\mathrm{e}^{-r}}{r}-1\right)^{2}, \quad r \geq 0 .
$$

It can be verified in a straightforward way that $\psi$ is a $C^{\infty}$ function, strictly increasing in $[0,+\infty), \psi(0)=0$ and $\psi([0,+\infty)) \subset[0,1)$. Taking into account these properties, we deduce that each term of the series (3.8) converges to zero when $\delta \rightarrow 0^{+}$and the whole series is bounded by $2 \sum_{k=1}^{\infty} a_{k}^{2}$, that is convergent because $y_{d} \in L_{+}^{2}(0,1)$. Thus, we conclude that tends to zero as $\delta \rightarrow 0^{+}$by using the Dominated Convergence Theorem for series.

This ends the proof of Theorem 2.2, but for proving later Theorem 2.3 corresponding to system (S1), it is convenient to make a small modification to previous construction as follows: instead of the control $u_{\delta}(x, t)$ given by (3.5), we can take a control with value zero at the end of the process. More precisely, we can consider for instance

$$
\hat{u}_{\delta}(x, t)= \begin{cases}0, & t \in(0, T-2 \delta), \\ \frac{1}{\delta} y_{d}(x), & t \in[T-2 \delta, T-\delta), \\ 0, & t \in[T-\delta, T]\end{cases}
$$


Applying the method of separation of variables in each time interval, it is not difficult to see here that the solution of $(S 1)$ with $y_{0}=0$ is given by

$$
\tilde{y}_{0, \hat{u}_{\delta}, \omega}(x, t)= \begin{cases}0, & t \in[0, T-2 \delta], \\ \frac{2}{\delta} \sum_{k=1}^{\infty} \frac{1-\mathrm{e}^{-\pi^{2} k^{2}(t-T+2 \delta)}}{\pi^{2} k^{2}} a_{k} \sin (k \pi x), & t \in[T-2 \delta, T-\delta], \\ \frac{2}{\delta} \sum_{k=1}^{\infty} \frac{1-\mathrm{e}^{-\pi^{2} k^{2} \delta}}{\pi^{2} k^{2}} \mathrm{e}^{-\pi^{2} k^{2}(t-T+\delta)} a_{k} \sin (k \pi x), & t \in[T-\delta, T],\end{cases}
$$

and therefore, arguing as before, we arrive to

$$
\left\|\tilde{y}_{0, \hat{u}_{\delta}, \omega}(\cdot, T)-y_{d}\right\|_{L^{2}(0,1)}^{2}=2 \sum_{k=1}^{\infty} a_{k}^{2}\left(\frac{\mathrm{e}^{-\pi^{2} k^{2} \delta}-\mathrm{e}^{-2 \pi^{2} k^{2} \delta}}{\pi^{2} k^{2} \delta}-1\right)^{2} .
$$

We can finish as above, seeing that the right-hand side of (3.11) tends to zero as $\delta \rightarrow 0^{+}$with the help of the new auxiliary function

$$
\hat{\psi}(r)=\left(\frac{\mathrm{e}^{-r}-\mathrm{e}^{-2 r}}{r}-1\right)^{2}, \quad r \geq 0,
$$

that satisfies the same properties than $\psi$.

\subsection{Proof of Theorem 2.4}

By the linearity of the problem, it is well known that $y_{y_{0}, u_{0}, u_{1}}=\tilde{y}_{y_{0}}+y_{0, u_{0}, u_{1}}$, and the following relation holds

$$
F_{y_{0}, b}(T)=\tilde{y}_{y_{0}}(\cdot, T)+F_{0, b}(T) .
$$

Therefore, the conclusion of Theorem 2.4 is equivalent to say that $F_{0, b}(T)$ is not dense in $L_{+}^{2}(0,1)$ and this is what we will prove.

At this point, let us remind that (in general) the solution $y_{0, u_{0}, u_{1}}$ must be defined by the transposition method (see [22], pp. 194-197). In our situation, its expression is given by

$$
y_{0, u_{0}, u_{1}}(x, t)=\sum_{k=1}^{\infty} b_{k}(t) \sqrt{2} \sin (k \pi x)
$$

where

$$
b_{k}(t)=\sqrt{2} k \pi \int_{0}^{t} \mathrm{e}^{-k^{2} \pi^{2}(t-s)}\left(u_{0}(s)+(-1)^{k+1} u_{1}(s)\right) \mathrm{d} s .
$$

It is not obvious at all that $y_{0, u_{0}, u_{1}}(\cdot, T) \in L^{2}(0,1)$. In fact, in [22], page 202, it is constructed an example where $y_{0, u_{0}, u_{1}}(\cdot, T) \notin L^{2}(0,1)$, by taking $u_{0}(t)=0$ and $u_{1}(t)=\frac{1}{\sqrt[4]{T-t}}$. The difficulty here is that $u_{1} \in L^{2}(0, T)$, but not in $L^{\infty}(0, T)$. In our case, using (3.13)-(3.14), we can estimate

$$
\int_{0}^{1}\left|y_{0, u_{0}, u_{1}}(x, T)\right|^{2} \mathrm{~d} x=\sum_{k=1}^{\infty}\left|b_{k}(T)\right|^{2} \leq C_{1} \sum_{k=1}^{\infty} \frac{1}{k^{2}}<\infty,
$$

with $C_{1}=\frac{2}{\pi^{2}}\left(\left\|u_{0}\right\|_{L^{\infty}(0, T)}+\left\|u_{1}\right\|_{L^{\infty}(0, T)}\right)^{2}$.

Moreover, applying the Theorem 2.5-(i) we derive that $y_{0, u_{0}, u_{1}}(x, T) \geq 0$, a.e. $x \in(0,1)$. Consequently, $F_{0, b}(T)$ is included in $L_{+}^{2}(0,1)$. Let us show that it is not dense in $L_{+}^{2}(0,1)$, arguing by contradiction. To this end, it can be checked by direct calculation that

$$
p(x, t)=-\exp \left(9 \pi^{2}(t-T)\right) \sin (3 \pi x)
$$


is the unique classical solution of the adjoint problem

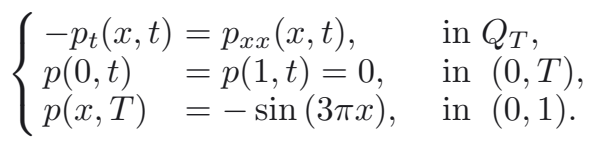

Moreover, it satisfies $p_{x}(0, t)<0, p_{x}(1, t)>0$ in $(0, T)$. Now, we select

$$
y_{d}(x)=\max \{p(x, T), 0\}, \quad x \in(0,1),
$$

as the target state in $L_{+}^{2}(0,1)$.

If $F_{0, b}(T)$ is dense in $L_{+}^{2}(0,1)$, there exist two sequences $\left\{u_{0 k}\right\}_{k=1}^{\infty},\left\{u_{1 k}\right\}_{k=1}^{\infty}$ in $L_{+}^{\infty}(0, T)$ such that for the corresponding solutions $\left\{y_{k}\right\}_{k=1}^{\infty}$ of $(S 3)$ with $y_{0}=0$ (namely, $y_{k}=y_{0, u_{0 k}, u_{1 k}}$ ), we have:

$$
y_{k}(\cdot, T) \longrightarrow y_{d} \quad \text { in } L_{+}^{2}(0,1) \text { as } k \rightarrow+\infty .
$$

Multiplying by $p$ the PDE satisfied by $y_{k}$ and integrating by parts, we get:

$$
\int_{0}^{1} y_{k}(x, T) p(x, T) \mathrm{d} x=\int_{0}^{T} u_{0 k}(t) p_{x}(0, t) \mathrm{d} t-\int_{0}^{T} u_{1 k}(t) p_{x}(1, t) \mathrm{d} t \leq 0 .
$$

(Alternatively, previous equality can be derived from the expressions (3.13)-(3.14) for each $y_{k}$.) Thus, we arrive to the contradiction

$$
\int_{1 / 3}^{2 / 3}(\sin (3 \pi x))^{2} \mathrm{~d} x=\int_{0}^{1} y_{d}(x) p(x, T) \mathrm{d} x=\lim _{k \rightarrow \infty} \int_{0}^{1} y_{k}(x, T) p(x, T) \mathrm{d} x \leq 0 .
$$

\subsection{Proof of Theorem 2.1 for system (S2)}

First, let us consider the case where $y_{0} \in C_{+}[0,1]$ having compact support in $(0,1)$. Let us fix $(\alpha, \beta)$ any subinterval of $(0,1) \backslash \omega$. By Theorem 2.4 corresponding to this interval $(\alpha, \beta)$, instead of $(0,1)$, we can pick out $y_{d} \in \tilde{y}_{y_{0}}(\cdot, T)+L_{+}^{2}(\alpha, \beta)$ such that $y_{d}$ does not belong to the closure of $F_{y_{0}, b}(T)$. We extend it by zero to the whole interval $(0,1)$, denoting this extension by $\tilde{y}_{d}$.

Arguing by contradiction, let us assume that there exist $\left\{v_{k}\right\}_{k=1}^{\infty} \subset L^{\infty}\left(Q_{T}\right)$ such that

$$
y_{y_{0}, v_{k}, \omega}(\cdot, T) \rightarrow \tilde{y}_{d} \quad \text { in } L_{+}^{2}(0,1) \text { as } k \rightarrow+\infty .
$$

Taking into account some classical regularity results (see [19], Thm. 9.1 and Cor., pp. 341-342) we know that $y_{y_{0}, v_{k}, \omega} \in C\left(\overline{Q_{T}}\right)$. Furthermore, $y_{y_{0}, v_{k}, \omega}$ is nonnegative thanks to the Theorem 2.5-(i). Consequently, the restriction of $y_{y_{0}, v_{k}, \omega}$ to the interval $(\alpha, \beta)$ can be viewed as the solution of one system of the type

$$
\begin{cases}y_{t}(x, t)=y_{x x}(x, t), & \text { in }(\alpha, \beta) \times(0, T), \\ y(\alpha, t)=u_{0}(t), & \text { in }(0, T), \\ y(\beta, t)=u_{1}(t), & \text { in }(0, T), \\ y(x, 0)=y_{0}(x), & \text { in }(\alpha, \beta),\end{cases}
$$

with nonnegative continuous boundary values at the extremes $\alpha$ and $\beta$. The contradiction arrives due to the choice of $y_{d}$ and the fact

$$
y_{y_{0}, v_{k}, \omega}(\cdot, T)_{\left.\right|_{(\alpha, \beta)}} \rightarrow y_{d} \quad \text { in } L_{+}^{2}(\alpha, \beta) \text { as } k \rightarrow+\infty .
$$

For the general case $y_{0} \in L_{+}^{2}(0,1)$, we can construct an approximating sequence $\left\{y_{0 k}\right\}_{k=1}^{\infty} \subset C_{+}[0,1]$ with compact support in $(0,1)$ such that $y_{0 k} \rightarrow y_{0}$ in $L^{2}(0,1)$, as $k \rightarrow+\infty$. The argumentation follows from the continuous dependence of the solution with respect to the initial datum, see for instance [19], Theorem 4.5, page 166 . 


\section{Proof of Theorem 2.3}

\subsection{Proof of Theorem 2.3 for system $(S 1)$}

To show that $F_{1,0, m b}^{+}(T)$ is dense in $L_{+}^{2}(0,1)$, we will use the technique developed in the proof of Theorem 2.2. First, let us fix $M$ the smallest natural number satisfying $M \cdot l \geq 1$, where $l$ is the (fixed) length of the mobile subinterval $\omega(t)$ (see (1.1)).

Given any element $y_{d} \in L_{+}^{2}(0,1)$, we can decompose it into $M$ "pieces" localized in disjoint subintervals of length (at most) $l$, as follows:

$$
\begin{gathered}
y_{d_{1}}(x)= \begin{cases}y_{d}(x), & x \in(0, l), \\
0, & x \in(l, 1),\end{cases} \\
y_{d_{j}}(x)= \begin{cases}y_{d}(x), & x \in((j-1) l, l j), \\
0, & \text { otherwise, }\end{cases}
\end{gathered}
$$

for $j=2, \ldots, M-1$

$$
y_{d_{M}}(x)= \begin{cases}y_{d}(x), & x \in((M-1) l, 1) \\ 0, & \text { otherwise }\end{cases}
$$

Clearly, each $y_{d j} \in L_{+}^{2}(0,1)$ and

$$
y_{d}(x)=\sum_{j=1}^{M} y_{d_{j}}(x), \quad \text { a.e. } x \in(0,1) .
$$

Moreover, given $\epsilon>0$, there exist $\hat{y}_{d_{j}} \in H_{0}^{1}(0,1) \cap L_{+}^{2}(0,1)$ for $j=1, \ldots, M$, with its support $\omega_{j}$ contained in the corresponding one of each $y_{d j}$ and verifying

$$
\sum_{j=1}^{M}\left\|\hat{y}_{d_{j}}-y_{d_{j}}\right\|_{L^{2}(0,1)} \leq \frac{\epsilon}{2} .
$$

Now, we can argue "by parts", using the constructions (3.9) and (3.5) established in the proof of Theorem 2.2, as follows: given $\hat{y}_{d_{1}}$, there exists $\delta_{1} \in\left(0, \frac{T}{2}\right)$ such that if we take the control

$$
\hat{u}_{1}(x, t)= \begin{cases}0, & t \in\left(0, T-2 \delta_{1}\right), \\ \frac{1}{\delta_{1}} \hat{y}_{d_{1}}(x), & t \in\left(T-2 \delta_{1}, T-\delta_{1}\right), \\ 0, & t \in\left(T-\delta_{1}, T\right),\end{cases}
$$

it is satisfied

$$
\left\|\tilde{y}_{0, \hat{u}_{1}, \omega_{1}}(\cdot, T)-\hat{y}_{d_{1}}\right\|_{L^{2}(0,1)} \leq \frac{\epsilon}{2 M} .
$$

Arguing now with $\hat{y}_{d_{2}}$, there exists $\delta_{2} \in\left(0, \frac{\delta_{1}}{2}\right)$ such that if we take the control

$$
\hat{u}_{2}(x, t)= \begin{cases}0, & t \in\left(0, T-2 \delta_{2}\right), \\ \frac{1}{\delta_{2}} \hat{y}_{d_{2}}(x), & t \in\left(T-2 \delta_{2}, T-\delta_{2}\right), \\ 0, & t \in\left(T-\delta_{2}, T\right),\end{cases}
$$

it is satisfied

$$
\left\|\tilde{y}_{0, \hat{u}_{2}, \omega_{2}}(\cdot, T)-\hat{y}_{d_{2}}\right\|_{L^{2}(0,1)} \leq \frac{\epsilon}{2 M} .
$$

Iterating this process, we obtain $\delta_{j} \in\left(0, \frac{\delta_{j-1}}{2}\right), j=1, \ldots, M$, and controls

$$
\hat{u}_{j}(x, t)= \begin{cases}0, & t \in\left(0, T-2 \delta_{j}\right), \\ \frac{1}{\delta_{j}} \hat{y}_{d_{j}}(x), & t \in\left(T-2 \delta_{j}, T-\delta_{j}\right), \\ 0, & t \in\left(T-\delta_{j}, T\right),\end{cases}
$$


$j=1, \ldots, M-1$, and

$$
\hat{u}_{M}(x, t)= \begin{cases}0, & t \in\left(0, T-\delta_{M}\right) \\ \frac{1}{\delta_{M}} \hat{y}_{d_{M}}(x), & t \in\left(T-\delta_{M}, T\right)\end{cases}
$$

for which

$$
\left\|\tilde{y}_{0, \hat{u}_{j}, \omega_{j}}(\cdot, T)-\hat{y}_{d_{j}}\right\|_{L^{2}(0,1)} \leq \frac{\epsilon}{2 M}, \quad j=1, \ldots, M .
$$

We finish the proof by taking

$$
u(x, t)=\sum_{j=1}^{M} \hat{u}_{j}(x, t), \quad \tilde{y}(x, t)=\sum_{j=1}^{M} \tilde{y}_{0, \hat{u}_{j}, \omega_{j}}(x, t)
$$

and noticing that by the linearity

$$
\begin{aligned}
\left\|\tilde{y}(\cdot, T)-y_{d}\right\|_{L^{2}(0,1)} & \leq\left\|\tilde{y}(\cdot, T)-\sum_{j=1}^{M} \hat{y}_{d_{j}}\right\|_{L^{2}(0,1)}+\left\|\sum_{j=1}^{M} \hat{y}_{d_{j}}-y_{d}\right\|_{L^{2}(0,1)} \\
& \leq \sum_{j=1}^{M}\left\|\tilde{y}_{0, \hat{u}_{j}, \omega_{j}}(\cdot, T)-\hat{y}_{d_{j}}\right\|_{L^{2}(0,1)}+\sum_{j=1}^{M}\left\|\hat{y}_{d_{j}}-y_{d_{j}}\right\|_{L^{2}(0,1)} \leq \epsilon .
\end{aligned}
$$

Let us remark that in each time subinterval $\left(T-\delta_{j}, T-\delta_{j+1}\right)$, the value of the control $u(x, t)$ coincides with the value of $\hat{u}_{j+1}(x, t)$ and hence its support is included in $\omega(t)=(r(t), r(t)+l)$, with $r(t) \in P C[0, T]$ given by

$$
r(t)=\left\{\begin{array}{lll}
0, & & t \in\left(0, T-\delta_{1}\right), \\
l, & & t \in\left(T-\delta_{1}, T-\delta_{2}\right), \\
2 l, & & t \in\left(T-\delta_{2}, T-\delta_{3}\right), \\
& \vdots & \\
j l, & & t \in\left(T-\delta_{j}, T-\delta_{j+1}\right), \\
& \vdots & \\
(M-2) l, & t \in\left(T-\delta_{M-2}, T-\delta_{M-1}\right), \\
1-l, & & t \in\left(T-\delta_{M-1}, T\right) .
\end{array}\right.
$$

This implies that $u(x, t)=u(x, t) \chi_{\omega(t)}(x)$ and $\tilde{y}=\tilde{y}_{0, u, r}$, as desired.

\subsection{Proof of Theorem 2.3 for system $(S 2)$}

We will prove the result in two steps. First, following the notations of Section 1 (compare with (1.2)), we introduce the set

$$
F_{2, y_{0}, m b}^{+}(T)=\left\{y_{y_{0}, v, r}(x, T): x \in(0,1), v \in L_{+}^{\infty}\left(Q_{T}\right) \text { and } r \in P C[0, T]\right\} .
$$

Applying twice the Theorem 2.5-(i) (first to $y_{y_{0}, v, r}$ and later to the difference $y_{y_{0}, v, r}-\tilde{y}_{y_{0}}$, treating the bilinear term as a free one), it is well known that

$$
y_{y_{0}, v, r}(x, T) \geq \tilde{y}_{y_{0}}(x, T), \text { a.e. } x \in(0,1), \forall v \in L_{+}^{\infty}\left(Q_{T}\right), \forall r \in P C[0, T] .
$$

\subsubsection{Step 1}

We will prove that the system $(S 2)$ is approximately nonnegative controllable at time $T$, in the following sense:

Theorem 4.1 (Nonnegative multiplicative mobile control). Let us assume that $y_{0} \in L_{+}^{2}(0,1), y_{0} \not \equiv 0$. Then, for any $T>0$, the set $F_{2, y_{0}, m b}^{+}(T)$ is dense in $\tilde{y}_{y_{0}}(\cdot, T)+L_{+}^{2}(0,1)$. 
Proof of Theorem 4.1. Pick out a generic element $y_{d} \in L_{+}^{2}(0,1)$. As a consequence of Theorem 2.3 for system $(S 1)$, given $\epsilon>0$, there exist $u \in L_{+}^{2}\left(Q_{T}\right)$ and $r \in P C[0, T]$ such that

$$
\left\|\tilde{y}_{0, u, r}(\cdot, T)-y_{d}\right\|_{L^{2}(0,1)}<\epsilon .
$$

By well known density results, we can suppose, without loss of generality, that $u \in C_{+}\left(\overline{Q_{T}}\right)$ (that is, $u$ is continuous and nonnegative in $\left.\overline{Q_{T}}\right)$ with compact support $K$ included in $(0,1) \times(\delta, T) \subset Q_{T}$, for some $\delta>0$.

Using classical regularity results (see [12], Thm. 10.2, p. 140, and [19], Thm. 9.1, pp. 341-342), it is also known that $\tilde{y}_{y_{0}} \in C^{\infty}\left(Q_{T}\right) \cap C_{+}([0,1] \times[\delta, T])$.

Now, let us consider $y=\tilde{y}_{y_{0}, u, r}$ and

$$
v(x, t)=\left\{\begin{array}{cl}
\frac{u(x, t)}{y(x, t)}, & (x, t) \in K \\
0, & (x, t) \in Q_{T} \backslash K
\end{array}\right.
$$

By the Theorem 2.5-(i), it is clear that $y \geq 0$, a.e. in $Q_{T}$. We will prove below that $v \in L_{+}^{\infty}\left(Q_{T}\right)$. Using this fact, it is straightforward to see that $y=y_{y_{0}, v, r}$ (i.e. $y$ is the solution of system (S2) corresponding to $\left.y_{0}, v, r\right)$, because $u(x, t) \chi_{\omega(t)}(x)=v(x, t) y(x, t) \chi_{\omega(t)}(x)$ in $Q_{T}$, and

$$
\begin{aligned}
\left\|y_{y_{0}, v, r}(\cdot, T)-\left(\tilde{y}_{y_{0}}(\cdot, T)+y_{d}\right)\right\|_{L^{2}(0,1)} & =\left\|\tilde{y}_{y_{0}, u, r}(\cdot, T)-\left(\tilde{y}_{y_{0}}(\cdot, T)+y_{d}\right)\right\|_{L^{2}(0,1)} \\
& =\left\|\tilde{y}_{0, u, r}(\cdot, T)-y_{d}\right\|_{L^{2}(0,1)}<\epsilon,
\end{aligned}
$$

as we were looking for.

Hence, it remains to show that $v \in L_{+}^{\infty}\left(Q_{T}\right)$. Since $\tilde{y}_{y_{0}} \in C_{+}([0,1] \times[\delta, T]), \tilde{y}_{y_{0}} \geq 0$ in $Q_{T}$ and $K$ is a compact set, there is a constant $\rho \geq 0$ such that $\tilde{y}_{y_{0}} \geq \rho$ in $K$. In fact, we can guarantee that $\rho>0$ : if there exists $\left(x_{1}, t_{1}\right) \in K \subset(0,1) \times(\delta, T)$ such that $\tilde{y}_{y_{0}}\left(x_{1}, t_{1}\right)=\rho=0$, thanks to the Theorem 2.5-(iii), we conclude that $\tilde{y}_{y_{0}} \equiv 0$ in $[0,1] \times\left[0, t_{1}\right]$ and, consequently, $y_{0} \equiv 0$ in $(0,1)$, which it is excluded by hypothesis. Then, we get

$$
\|v\|_{L^{\infty}\left(Q_{T}\right)} \leq \frac{\|u\|_{L^{\infty}(K)}}{\rho}
$$

\subsubsection{Step 2}

We want to approximate any element $y_{d} \in L_{+}^{2}(0,1)$, even if $y_{d} \notin \tilde{y}_{y_{0}}(\cdot, T)+L_{+}^{2}(0,1)$. Roughly speaking, the main idea here is to take very large negative controls at the beginning of the process and move the control support across the space domain, in order to reduce the lower barrier that represents $\tilde{y}_{y_{0}}(\cdot, T)$, making it small enough.

For that purpose, we fix $M$ (as previously) the smallest natural number satisfying $M \cdot l \geq 1$, where $l$ is the length of the mobile subinterval $\omega(t)$. Given some intermediate times $0 \leq T_{1} \leq T_{2} \leq \ldots \leq T_{M}<T$, let us denote by $y_{1}(x, t)$ the unique solution of the following system

$$
\begin{cases}y_{t}(x, t)=y_{x x}(x, t)-m_{1} y(x, t) \chi_{(0, l)}(x), & \text { in }(0,1) \times\left(0, T_{1}\right), \\ y(0, t)=y(1, t)=0, & \text { in }\left(0, T_{1}\right) \\ y(x, 0)=y_{0}(x), & \text { in }(0,1)\end{cases}
$$

where $m_{1} \geq 0$ will be carefully chosen later. Assuming now that $y_{j-1}$ is already known, $y_{j}$ will denote the solution of the system

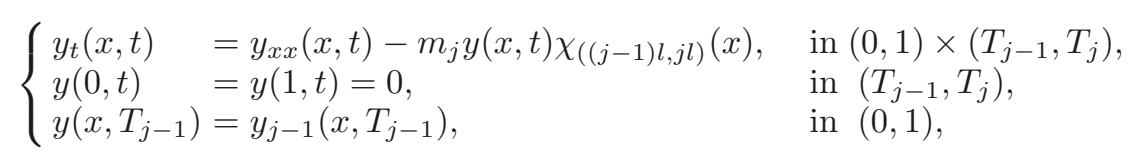


with $m_{j} \geq 0$ for $j=1, \ldots, M-1$ also to be chosen. Finally, $y_{M}$ will denote the solution of the problem

$$
\left\{\begin{array}{lll}
y_{t}(x, t)=y_{x x}(x, t)-m_{M} y(x, t) \chi_{((M-1) l, 1)}(x), & & \text { in }(0,1) \times\left(T_{M-1}, T_{M}\right), \\
y(0, t) & =y(1, t)=0, & \text { in }\left(T_{M-1}, T_{M}\right), \\
y\left(x, T_{M-1}\right)=y_{M-1}\left(x, T_{M-1}\right), & \text { in }(0,1),
\end{array}\right.
$$

with $m_{M} \geq 0$ to be chosen. The following result will be essential in this section:

Theorem 4.2. With previous notations, given $\epsilon>0$, there exist nonnegative constants $m_{1}, \ldots, m_{M}$ and intermediate times $0 \leq T_{1} \leq T_{2} \leq \ldots \leq T_{M}<T$ such that

$$
\left\|y_{M}\left(\cdot, T_{M}\right)\right\|_{L^{2}(0,1)} \leq \frac{\epsilon}{2}
$$

We postpone its proof to the next sections, but we will conclude now the proof of Theorem 2.3. Given $\hat{y}_{1}(x, t)$ the unique solution of the system

$$
\begin{cases}y_{t}(x, t)=y_{x x}(x, t), & \text { in }(0,1) \times\left(T_{M}, T\right) \\ y(0, t)=y(1, t)=0, & \text { in }\left(T_{M}, T\right) \\ y\left(x, T_{M}\right)=y_{M}\left(x, T_{M}\right), & \text { in }(0,1)\end{cases}
$$

it is well known that

$$
\left\|\hat{y}_{1}(\cdot, T)\right\|_{L^{2}(0,1)} \leq\left\|y_{M}\left(\cdot, T_{M}\right)\right\|_{L^{2}(0,1)} \leq \frac{\epsilon}{2},
$$

where the last inequality comes from Theorem 4.2 .

Thanks to the Theorem 2.5-(i), it holds that $y_{j}(x, t) \geq 0$ a.e. $(x, t) \in Q_{T}$ for $j=1, \ldots, M$. In particular, $y_{M}\left(x, T_{M}\right) \geq 0$ a.e. $x \in(0,1)$.

Assume that $y_{d} \in L_{+}^{2}(0,1)$ and $\epsilon>0$ are fixed. Applying now Theorem 4.1 for the corresponding system of type $(S 2)$ in the domain $(0,1) \times\left(T_{M}, T\right)$ with initial datum $y_{M}\left(\cdot, T_{M}\right)$, we deduce the existence of $v_{2} \in$ $L_{+}^{\infty}\left((0,1) \times\left(T_{M}, T\right)\right)$ and $r_{2} \in P C\left[T_{M}, T\right]$ such that the solution $\hat{y}_{2}(x, t)$ of the system

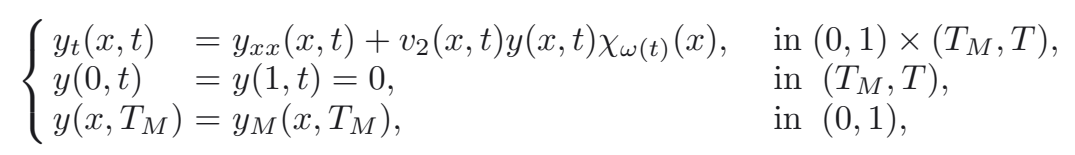

satisfies

$$
\left\|\hat{y}_{2}(\cdot, T)-\left(y_{d}+\hat{y}_{1}(\cdot, T)\right)\right\|_{L^{2}(0,1)}<\frac{\epsilon}{2} .
$$

We finish the proof, by noticing that

$$
y_{y_{0}, v, r}(x, t)= \begin{cases}\hat{y}_{1}(x, t), & (x, t) \in(0,1) \times\left(0, T_{M}\right] \\ \hat{y}_{2}(x, t), & (x, t) \in(0,1) \times\left[T_{M}, T\right)\end{cases}
$$

with

$$
v(x, t)=\left\{\begin{array}{cll}
-m_{1}, & & (x, t) \in(0,1) \times\left(0, T_{1}\right), \\
-m_{2}, & & (x, t) \in(0,1) \times\left(T_{1}, T_{2}\right), \\
& \vdots & \\
-m_{j}, & & (x, t) \in(0,1) \times\left(T_{j-1}, T_{j}\right), \\
& \vdots & \\
-m_{M}, & (x, t) \in(0,1) \times\left(T_{M-1}, T_{M}\right), \\
v_{2}(x, t), & (x, t) \in(0,1) \times\left(T_{M}, T\right),
\end{array}\right.
$$


and

$$
r(t)=\left\{\begin{array}{lll}
0, & & t \in\left(0, T_{1}\right), \\
l, & & t \in\left(T_{1}, T_{2}\right), \\
& \vdots & \\
& & t \in\left(T_{j}, T_{j+1}\right), \\
& \vdots & \\
(M-2) l, & t \in\left(T_{M-2}, T_{M-1}\right), \\
1-l, & & t \in\left(T_{M-1}, T_{M}\right), \\
r_{2}(t), & & t \in\left(T_{M}, T\right) .
\end{array}\right.
$$

Clearly, $v \in L^{\infty}\left(Q_{T}\right)$ and $r \in P C[0, T]$. Moreover, thanks to (4.21) and (4.23), it is satisfied

$$
\begin{aligned}
\left\|y_{y_{0}, v, r}(\cdot, T)-y_{d}\right\|_{L^{2}(0,1)} & =\left\|y_{y_{0}, v, r}(\cdot, T)-\left(y_{d}+\hat{y}_{1}(\cdot, T)\right)+\hat{y}_{1}(\cdot, T)\right\|_{L^{2}(0,1)} \\
& \leq\left\|y_{y_{0}, v, r}(\cdot, T)-\left(y_{d}+\hat{y}_{1}(\cdot, T)\right)\right\|_{L^{2}(0,1)}+\left\|\hat{y}_{1}(\cdot, T)\right\|_{L^{2}(0,1)} \\
& =\left\|\hat{y}_{2}(\cdot, T)-\left(y_{d}+\hat{y}_{1}(\cdot, T)\right)\right\|_{L^{2}(0,1)}+\left\|\hat{y}_{1}(\cdot, T)\right\|_{L^{2}(0,1)}<\epsilon,
\end{aligned}
$$

as we were looking for.

\subsubsection{Auxiliary results}

Our proof for Theorem 4.2 is quite technical and relies on the following results:

Proposition 4.3. Assume that $v \in C[0,1]$ with $v(x) \leq 0$ for all $x \in[0,1]$ and $y_{0} \in C^{2+\sigma}[0,1]$, with $\sigma \in(0,1)$ verifies $y_{0}(0)=y_{0}(1)=y_{0}^{\prime \prime}(0)=y_{0}^{\prime \prime}(1)=0$ and $y_{0}(x) \geq 0$ for all $x \in[0,1]$. Then, the classical solution of the problem

$$
\begin{cases}y_{t}(x, t)=y_{x x}(x, t)+v(x) y(x, t), & \text { in } Q_{T}, \\ y(0, t)=y(1, t)=0, & \text { in }(0, T), \\ y(x, 0)=y_{0}(x), & \text { in }(0,1),\end{cases}
$$

satisfies the following properties:

(a) $y_{t}(x, t) \leq \max _{x \in[0,1]}\left(y_{0}^{\prime \prime}(x)\right)^{+}, \quad \forall(x, t) \in \overline{Q_{T}}$,

(b) $0 \leq y_{x}(0, t) \leq e \cdot \max _{x \in[0,1]}\left(y_{0}^{\prime}(x) \mathrm{e}^{y_{0}(x)}\right), \quad \forall t \in[0, T]$ and

(c) $e \cdot \min _{x \in[0,1]}\left(y_{0}^{\prime}(x) \mathrm{e}^{y_{0}(x)}\right) \leq y_{x}(1, t) \leq 0, \quad \forall t \in[0, T]$.

Proof of Proposition 4.3. In the case $y_{0} \equiv 0$ the result is clearly valid, because $y \equiv 0$. So, let us suppose $y_{0} \not \equiv 0$.

The existence and uniqueness of a classical solution $y \in C^{2+\sigma, 1+\sigma / 2}\left(\overline{Q_{T}}\right)$ for problem (4.28) is a consequence of [19], Theorem 5.2, page 320. Moreover, by the Theorem 2.5-(i) and (ii), it is also known that

$$
0 \leq y(x, t) \leq\left\|y_{0}\right\|_{L^{\infty}(0,1)} \forall(x, t) \in \overline{Q_{T}} .
$$

To prove $(a)$, we differentiate (4.28) with respect to the time variable to deduce that $y_{t}$ is the solution of the problem

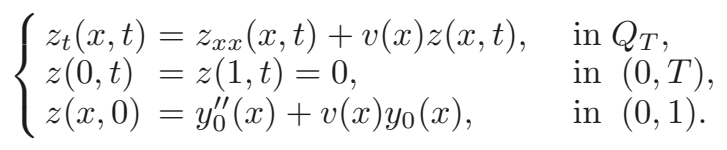

Using once more the Theorem 2.5-(ii) and taking into account that $v$ is nonpositive and $y_{0}$ is nonnegative in $[0,1]$, we get

$$
y_{t}(x, t) \leq \max _{x \in[0,1]}\left(y_{0}^{\prime \prime}(x)+v(x) y_{0}(x)\right)^{+} \leq \max _{x \in[0,1]}\left(y_{0}^{\prime \prime}(x)\right)^{+} \quad \forall(x, t) \in \overline{Q_{T}} .
$$


The lower bound of $b$ ) is a direct consequence of the definition, the homogeneous boundary condition and that $y$ is nonnegative. To derive the upper bound, we will use Bernstein's method (see [19], pp. 414 and 537), by introducing the auxiliary function $w(x, t)=\mathrm{e}^{y(x, t)}+\rho \mathrm{e}^{1-x}-1$ with $\rho=\max _{x \in[0,1]}\left(y_{0}^{\prime}(x) \mathrm{e}^{y_{0}(x)}\right)$. Let us remark that $\rho>0$, thanks to the hypotheses on $y_{0}$.

By direct calculation it can checked that $w \in C^{2,1}\left(\overline{Q_{T}}\right)$ and satisfies the following PDE:

$$
w_{t}(x, t)-w_{x x}(x, t)=\left(v(x) y(x, t)-\left(y_{x}(x, t)\right)^{2}\right) \mathrm{e}^{y(x, t)}-\rho \mathrm{e}^{1-x} .
$$

Let us denote by $\left(x_{0}, t_{0}\right)$ a point in $\overline{Q_{T}}$ where $w$ takes its maximum value. We will see that $x_{0}=0$. If $\left(x_{0}, t_{0}\right) \in$ $Q_{T}$, it is well known that $w_{t}\left(x_{0}, t_{0}\right)=0$ and $w_{x x}\left(x_{0}, t_{0}\right) \leq 0$, and using the previous PDE we arrive to the contradiction

$$
0 \leq w_{t}\left(x_{0}, t_{0}\right)-w_{x x}\left(x_{0}, t_{0}\right) \leq-\rho \mathrm{e}^{1-x_{0}}<0 .
$$

The same argumentation can be used to exclude the case $t_{0}=T$, because the unique difference is that $w_{t}\left(x_{0}, t_{0}\right) \geq 0$. Consequently, $\left(x_{0}, t_{0}\right)$ must be a point of the "parabolic" boundary of $Q_{T}$, that is, $x_{0}=0$, $x_{0}=1$ or $t_{0}=0$. The last two possibilities can be excluded by noticing that $w(0, t)=\rho e \geq \rho=w(1, t)$, $w(x, 0)=\mathrm{e}^{y_{0}(x)}+\rho \mathrm{e}^{1-x}-1$, and taking into account the choice for $\rho$ :

$$
w_{x}(x, 0)=y_{0}^{\prime}(x) \mathrm{e}^{y_{0}(x)}-\rho \mathrm{e}^{1-x} \leq \rho-\rho \mathrm{e}^{1-x} \leq 0, \quad \forall x \in[0,1] .
$$

Therefore, $w(x, 0)$ is non-increasing in $[0,1]$ and $w(x, 0) \leq w(0,0)=\rho e$, for all $x \in[0,1]$. Consequently, $w(x, t) \leq \rho e=w(0, t)$, for all $(x, t) \in \overline{Q_{T}}$ and hence, $w_{x}(0, t) \leq 0$ for all $t \in[0,1]$. This is equivalent to say that $y_{x}(0, t) \leq \rho e$ for all $t \in[0,1]$.

The proof for $c$ ) is similar, using now the auxiliary function $\tilde{w}(x, t)=\mathrm{e}^{y(x, t)}-\tilde{\rho} \mathrm{e}^{x}-1$ with

$$
\tilde{\rho}=\min _{x \in[0,1]}\left(y_{0}^{\prime}(x) \mathrm{e}^{y_{0}(x)}\right)<0 .
$$

Corollary 4.4. Under the same hypotheses of Proposition 4.3, except that $v \in L^{\infty}(0,1)$, the conclusions $(a)-(c)$ remain valid, for almost every point.

Proof of Corollary 4.4. By the classical convolution technique, it can be deduced the existence of a sequence $\left\{v_{k}\right\}_{k=1}^{\infty} \subset C[0,1]$ such that $-\|v\|_{L^{\infty}(0,1)} \leq v_{k}(x) \leq 0$ for all $k=1,2, \ldots$, in $[0,1]$ and $v_{k} \rightarrow v$ in $L^{q}(0,1)$, as $k \rightarrow+\infty$, for some $q>2$. If we denote by $y_{v_{k}} \in C^{2,1}\left(\overline{Q_{T}}\right)$ the classical solution of problem (4.28) with $v=v_{k}$, applying [19], Theorem 9.1, page 341, to the difference $y_{v_{k}}-y$ and using that $0 \leq y_{v_{k}}(x, t) \leq\left\|y_{0}\right\|_{L^{\infty}(0,1)}$ in $Q_{T}$, by Theorem 2.5, we get

$$
\left\|y_{v_{k}}-y\right\|_{W_{q}^{2,1}\left(Q_{T}\right)} \leq C\left\|v_{k}-v\right\|_{L^{q}\left(Q_{T}\right)} \rightarrow 0 \quad \text { as } k \rightarrow+\infty .
$$

In particular,

$$
\left(y_{v_{k}}\right)_{t} \longrightarrow y_{t} \quad \text { in } L^{q}\left(Q_{T}\right), \text { as } k \rightarrow+\infty .
$$

Combining the estimate above with [19], Lemma 3.4, page 82, for the values $m=s=1, r=0$ and $q>2$, we deduce

$$
\left(y_{v_{k}}\right)_{x}(0, t) \longrightarrow y_{x}(0, t) \text { and }\left(y_{v_{k}}\right)_{x}(1, t) \longrightarrow y_{x}(1, t) \text { in } L^{q}(0, T), \text { as } k \rightarrow+\infty .
$$

Using now the corresponding expressions $(a)-(c)$ for each $y_{v_{k}}$ and the fact that the lower and upper bounds do not depend on $v_{k}$, we can pass to the limit in them and arrive to the same ones for $y$ that will be satisfied for almost every point. 


\subsubsection{Proof of Theorem 4.2}

The conclusion of the Theorem 4.2 is clearly valid if the $L^{2}$-norm of the initial datum is small enough compared with $\epsilon$. More precisely, if $\left\|y_{0}\right\|_{L^{2}(0,1)} \leq \frac{\epsilon}{2} \mathrm{e}^{T \pi^{2}}$, it is sufficient to take $T_{1}=T_{2}=\ldots=T_{M}=0$ and $m_{1}=m_{2}=\ldots=m_{M}=0$. Of course, we want to deal with the general case.

Let us begin with a useful observation. As we have mentioned before, it is well known that for each $y_{0} \in$ $L_{+}^{2}(0,1)$, we can construct an approximating sequence $\left\{y_{0 k}\right\}_{k=1}^{\infty} \subset C^{2+\sigma}[0,1]$ with compact support in $(0,1)$ such that $y_{0 k}(x) \geq 0$ for all $x \in[0,1]$ and $k$, with $y_{0 k} \rightarrow y_{0}$ in $L^{2}(0,1)$, as $k \rightarrow+\infty$. If we denote by $y_{y_{0}}$ the unique solution of (4.28) and by $y_{y_{0 k}}$ the unique solution of the same problem with initial datum $y_{0 k}$ instead of $y_{0}$, subtracting both PDE, multiplying it by the difference $y_{y_{0}}-y_{y_{0 k}}$ and integrating by parts, we arrive to the expression

$$
\begin{aligned}
\frac{1}{2} \int_{0}^{1}\left(y_{y_{0}}(x, T)-y_{y_{0 k}}(x, T)\right)^{2} \mathrm{~d} x-\frac{1}{2} \int_{0}^{1}\left(y_{0}(x)-y_{0 k}(x)\right)^{2} \mathrm{~d} x= & -\int_{Q_{T}}\left(\left(y_{y_{0}}(x, t)-y_{y_{0 k}}(x, t)\right)_{x}\right)^{2} \mathrm{~d} x \mathrm{~d} t \\
& +\int_{Q_{T}} v(x)\left(y_{y_{0}}(x, t)-y_{y_{0 k}}(x, t)\right)^{2} \mathrm{~d} x \mathrm{~d} t \leq 0,
\end{aligned}
$$

thanks to the hypothesis $v(x) \leq 0$, a.e. $x \in(0,1)$. Therefore,

$$
\int_{0}^{1}\left(y_{y_{0}}(x, T)-y_{y_{0 k}}(x, T)\right)^{2} \mathrm{~d} x \leq \int_{0}^{1}\left(y_{0}(x)-y_{0 k}(x)\right)^{2} \mathrm{~d} x,
$$

and, consequently, it is possible to make the $L^{2}$-norm of the difference of the solutions at time $T$ as small as needed, by taking the $L^{2}$-norm of the initial conditions small enough. As a consequence of this observation and taking into account the conclusion of the Theorem 4.2 that we want to derive, we will assume in the sequel that the initial data of the problems appearing along the proof are nonnegative regular functions with compact support in $(0,1)$.

Now, we are in conditions to prove the existence of the nonnegative constants $m_{1}, \ldots, m_{M}$, the intermediate times $0 \leq T_{1} \leq T_{2} \leq \ldots \leq T_{M}<T$ and the corresponding solutions $y_{j}$ for $j=1, \ldots, M$ (see (4.17)-(4.19)), such that the conclusion of the Theorem 4.2 holds.

For that purpose, we consider the family of problems

$$
\begin{cases}y_{t}(x, t)=y_{x x}(x, t)-m y(x, t) \chi_{(0, l)}(x), & \text { in } Q_{T} \\ y(0, t)=y(1, t)=0, & \text { in }(0, T), \\ y(x, 0)=y_{0}(x) & \text { in }(0,1)\end{cases}
$$

and denote its solution by $y_{m, 1}$ for each $m \geq 0$. Multiplying the PDE by $y_{m, 1}$ and integrating by parts in $Q_{T}$, we get as above

$$
\frac{1}{2} \int_{0}^{1}\left(y_{m, 1}(x, T)\right)^{2} \mathrm{~d} x-\frac{1}{2} \int_{0}^{1}\left(y_{0}(x)\right)^{2} \mathrm{~d} x=-\int_{Q_{T}}\left(\left(y_{m, 1}\right)_{x}(x, t)\right)^{2} \mathrm{~d} x \mathrm{~d} t-m \int_{0}^{T} \int_{0}^{l}\left(y_{m, 1}(x, t)\right)^{2} \mathrm{~d} x \mathrm{~d} t .
$$

Consequently,

$$
\int_{0}^{T} \int_{0}^{l}\left(y_{m, 1}(x, t)\right)^{2} \mathrm{~d} x \mathrm{~d} t \leq \frac{1}{2 m} \int_{0}^{1}\left(y_{0}(x)\right)^{2} \mathrm{~d} x .
$$

From this inequality we deduce that the sequence $g_{m}(t)=\sqrt{\int_{0}^{l}\left(y_{m, 1}(x, t)\right)^{2} \mathrm{~d} x}$ converges towards 0 as $m \rightarrow+\infty$ in $L^{2}(0, T)$ and (taking a subsequence, if necessary) we have that $g_{m}(t) \rightarrow 0$ as $m \rightarrow+\infty$ for a.e. $t \in(0, T)$. So, given $\epsilon>0$, there exists $T_{1} \in(0, T)$ and $m_{1} \gg 0$ such that $g_{m_{1}}\left(T_{1}\right)<\frac{\epsilon}{2 \sqrt{2 M-1}}$. Denoting $y_{1}=y_{m_{1}, 1}$ this is equivalent to say

$$
\int_{0}^{l}\left(y_{1}\left(x, T_{1}\right)\right)^{2} \mathrm{~d} x \leq \frac{\epsilon^{2}}{4(2 M-1)} .
$$


Following the same scheme, we consider now the family of problems

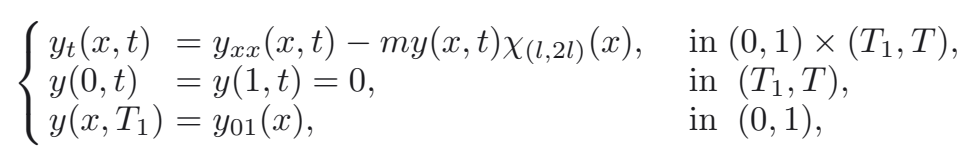

with $y_{01}(x)=y_{1}\left(x, T_{1}\right), y_{1}$ being the function obtained previously. If we denote its solution as $y_{m, 2}$ for each $m \geq 0$, repeating exactly the same argumentation, it is possible to derive the existence of $T_{2} \in\left(T_{1}, T\right)$ and $m_{2} \gg 0$ such that $y_{2}=y_{m_{2}, 2}$ satisfies

$$
\int_{l}^{2 l}\left(y_{2}\left(x, T_{2}\right)\right)^{2} \mathrm{~d} x \leq \frac{\epsilon^{2}}{4(2 M-1)} .
$$

In fact, we can select $T_{2}$ as close to $T_{1}$ as needed: more precisely, satisfying

$$
0<T_{2}-T_{1} \leq \min \left\{\frac{\epsilon^{2}}{8(2 M-1) C_{1}}, T-T_{1}\right\}
$$

with

$$
C_{1}=\left\|y_{01}\right\|_{L^{\infty}(0,1)}\left(l \cdot \max _{x \in[0,1]}\left(y_{01}^{\prime \prime}(x)\right)^{+}+e \cdot \max _{x \in[0,1]}\left(y_{01}^{\prime}(x) \mathrm{e}^{y_{01}(x)}\right)\right) .
$$

At this point it is evident that we are selecting the initial datum $y_{01}$ to be a $C^{2+\sigma}$ function, with $\sigma \in(0,1)$, with compact support in $(0,1)$ (see the beginning of this section). This is needed for the right-hand term of $(4.36)$ to be well defined. Let us remark that the terms appearing in (4.36) are clearly related with the conclusions of Proposition 4.3 (items $(a)-(b)$ ).

Under these conditions, we want to prove that

$$
\int_{0}^{2 l}\left(y_{2}\left(x, T_{2}\right)\right)^{2} \mathrm{~d} x \leq \frac{3 \epsilon^{2}}{4(2 M-1)} .
$$

Taking into account (4.34), this will be true if we derive

$$
\int_{0}^{l}\left(y_{2}\left(x, T_{2}\right)\right)^{2} \mathrm{~d} x \leq \frac{\epsilon^{2}}{2(2 M-1)} .
$$

This is the most delicate point of the proof, where Corollary 4.4 is essential. Again, we multiply the PDE of problem (4.33) with $m=m_{2}$ by $y_{2}$ and integrate by parts in the domain $(x, t) \in(0, l) \times\left(T_{1}, T_{2}\right)$, to obtain

$$
\frac{1}{2} \int_{0}^{l}\left(y_{2}\left(x, T_{2}\right)\right)^{2} \mathrm{~d} x-\frac{1}{2} \int_{0}^{l}\left(y_{01}(x)\right)^{2} \mathrm{~d} x=-\int_{T_{1}}^{T_{2}} \int_{0}^{l}\left(\left(y_{2}\right)_{x}(x, t)\right)^{2} \mathrm{~d} x \mathrm{~d} t+\int_{T_{1}}^{T_{2}}\left(y_{2}\right)_{x}(l, t) y_{2}(l, t) \mathrm{d} t,
$$

where the boundary condition at $x=0$ has been utilized together with the fact that $(0, l) \cap(l, 2 l)=\emptyset$. In particular, combining this relation with (4.32), we have

$$
\begin{aligned}
\int_{0}^{l}\left(y_{2}\left(x, T_{2}\right)\right)^{2} \mathrm{~d} x & \leq \int_{0}^{l}\left(y_{01}(x)\right)^{2} \mathrm{~d} x+2 \int_{T_{1}}^{T_{2}}\left(y_{2}\right)_{x}(l, t) y_{2}(l, t) \mathrm{d} t \\
& \leq \frac{\epsilon^{2}}{4(2 M-1)}+2 \int_{T_{1}}^{T_{2}}\left(y_{2}\right)_{x}(l, t) y_{2}(l, t) \mathrm{d} t,
\end{aligned}
$$

and the estimate (4.38) will hold, if we are able to prove that

$$
2 \int_{T_{1}}^{T_{2}}\left(y_{2}\right)_{x}(l, t) y_{2}(l, t) \mathrm{d} t \leq \frac{\epsilon^{2}}{4(2 M-1)} .
$$


Let us show that this holds thanks to the choice of $T_{2}$ (see (4.35)-(4.36)). Using Corollary 4.4 (with $v(x)=$ $-m_{2} \cdot \chi_{(l, 2 l)}(x)$ that is only a bounded function) for problem (4.33), it follows that

$$
\begin{aligned}
\left(y_{2}\right)_{x}(l, t) & =\left(y_{2}\right)_{x}(0, t)+\int_{0}^{l}\left(y_{2}\right)_{x x}(x, t) \mathrm{d} x=\left(y_{2}\right)_{x}(0, t)+\int_{0}^{l}\left(y_{2}\right)_{t}(x, t) \mathrm{d} x \\
& \leq e \cdot \max _{x \in[0,1]}\left(y_{01}^{\prime}(x) \mathrm{e}^{y_{01}(x)}\right)+l \cdot \max _{x \in[0,1]}\left(y_{01}^{\prime \prime}(x)\right)+\stackrel{\text { def }}{=} C_{2} .
\end{aligned}
$$

We can now combine this estimate with the fact that $0 \leq y_{2} \leq\left\|y_{01}\right\|_{L^{\infty}(0,1)}$ thanks to the Theorem 2.5-(i) and (ii), to get

$$
2 \int_{T_{1}}^{T_{2}}\left(y_{2}\right)_{x}(l, t) y_{2}(l, t) \mathrm{d} t \leq 2 \int_{T_{1}}^{T_{2}} C_{2} \cdot\left\|y_{01}\right\|_{L^{\infty}(0,1)} \mathrm{d} t=2\left(T_{2}-T_{1}\right) C_{1} \leq \frac{\epsilon^{2}}{4(2 M-1)},
$$

that is exactly (4.41).

Finally, we can repeat the argumentation to select the constant $m_{j}$, the time $T_{j} \in\left[T_{j-1}, T\right)$ and the solutions $y_{j}, j=1,2, \ldots, M-1$, verifying

$$
\int_{0}^{j l}\left(y_{j}\left(x, T_{j}\right)\right)^{2} \mathrm{~d} x \leq \frac{(2 j-1) \epsilon^{2}}{4(2 M-1)}
$$

and arrive to

$$
\int_{0}^{1}\left(y_{M}\left(x, T_{M}\right)\right)^{2} \mathrm{~d} x \leq \frac{\epsilon^{2}}{4}
$$

as desired.

\section{REFERENCES}

[1] A. Baciotti, Local Stabilizability of Nonlinear Control Systems. World Scientific, Singapore (1992).

[2] J.M. Ball and M. Slemrod, Feedback stabilization of distributed semilinear control systems. Appl. Math. Optim. 5 (1979) 169-179.

[3] J.M. Ball, J.E. Mardsen and M. Slemrod, Controllability for distributed bilinear systems. SIAM J. Control Optim. 20 (1982) $575-597$.

[4] L. Baudouin and J. Salomon, Constructive solution of a bilinear optimal control problem for a Schrödinger equation. Syst. Control Lett. 57 (2008) 453-464.

[5] K. Beauchard and C. Laurent, Local controllability of 1D linear and nonlinear Schrödinger equations with bilinear control. J. Math. Pures Appl. 94 (2010) 520-554.

[6] P. Cannarsa and A.Y. Khapalov, Multiplicative controllability for reaction-diffusion equations with target states admitting finitely many changes of sign. Discrete Contin. Dyn. Syst. Ser. B 14 (2010) 1293-1311.

[7] T. Chambrion, P. Mason, M. Sigalotti, and U. Boscain, Controllability of the discrete-spectrum Schrödinger equation driven by an external field. Ann. Inst. Henri Poincaré, Anal. Non Linéaire 26 (2009) 329-349.

[8] J.M. Coron, On the small-time local controllability of a quantum particle in a moving one-dimensional infinite square potential well. C. R. Math. Acad. Sci. Paris 342 (2006) 103-108.

[9] J.I. Díaz, J. Henry and A.M. Ramos, On the approximate controllability of some semilinear parabolic boundary-value problems. Appl. Math. Optim. 37 (1998) 71-97.

[10] S. Ervedoza and J.P. Puel, Approximate controllability for a system of Schrödinger equations modeling a single trapped ion. Ann. Inst. Henri Poincaré, Anal. Non Linéaire 26 (2009) 2111-2136.

[11] L.A. Fernández, Controllability of some semilinear parabolic problems with multiplicative control, presented at the Fifth SIAM Conference on Control and its applications, held in San Diego (2001).

[12] A. Friedman, Partial Differential Equations. Holt, Rinehart and Winston, New York (1969).

[13] A.Y. Khapalov, Mobile point controls versus locally distributed ones for the controllability of the semilinear parabolic equation. SIAM J. Control Optim. 40 (2001) 231-252.

[14] A.Y. Khapalov, Controllability of the semilinear parabolic equation governed by a multiplicative control in the reaction term: A qualitative approach. SIAM J. Control. Optim. 41 (2003) 1886-1900.

[15] A.Y. Khapalov, Controllability properties of a vibrating string with variable axial load. Discrete Contin. Dyn. Syst. 11 (2004) 311-324.

[16] A.Y. Khapalov, Controllability of Partial Differential Equations Governed by Multiplicative Controls, edited by Springer Verlag. Lect. Notes Math. 1995 (2010). 
[17] A.Y. Khapalov and R.R. Mohler, Reachable sets and controllability of bilinear time-invariant systems: A qualitative approach. IEEE Trans. Automat. Control 41 (1996) 1342-1346.

[18] K. Kime, Simultaneous control of a rod equation and a simple Schrödinger equation. Syst. Control Lett. 24 (1995) 301-306.

[19] O.A. Ladyzhenskaya, V.A. Solonnikov and N.N. Ural'tseva, Linear and Quasilinear Equations of Parabolic Type. Am. Math. Soc., Providence, RI (1968).

[20] S. Lenhart and M. Liang, Bilinear optimal control for a wave equation with viscous damping. Houston J. Math. 26 (2000) $575-595$.

[21] M. Liang, Bilinear optimal control for a wave equation. Math. Models Methods Appl. Sci. 9 (1999) 45-68.

[22] J.L. Lions, Optimal Control of Systems Governed by Partial Differential Equations. Springer-Verlag (1971).

[23] S. Müller, Strong convergence and arbitrarily slow decay of energy for a class of bilinear control problems. J. Differ. Equ. 81 (1989) 50-67.

[24] A.I. Prilepko, D.G. Orlovsky and I.A. Vasin, Methods for solving inverse problems in mathematical physics. Marcel Dekker Inc., New York (2000).

[25] R. Rink and R.R. Mohler, Completely controllable bilinear systems. SIAM J. Control 6 (1968) 477-486. 